\title{
Bio-efficacy and Phytotoxicity of Oxyfluorfen 2.5\% + Glyphosate 41\% SC (Ready mix) against Weed Complex in Grape
}

\author{
S. S. Nooli*, M. B. Patil and Nilesh Shiragavi \\ ACRP on Sugarcane, ARS, Sankeshwar, Karnataka, India \\ *Corresponding author
}

\section{A B S T R A C T}

\begin{abstract}
A field experiments was conducted on grape (Vitis vinifera L. cv Thompson seedless at the vineyard of the farmer's field of Vijayapur a district coming under the jurdiction of University of Agricultural Sciences Dharwad, Karnataka, after October pruning during 2014-15 and 2015-16 to study the bioefficacy of oxyfluorfen $2.5 \%+$ glyphosate $41 \%$ SC against weed complex. The weed density and weed dry weight recorded at 45 and 60 days after application (DAA) revealed that the dosage of the new herbicide oxyfluorfen $2.5 \%+$ glyphosate $41 \%$ SC @ 3.6251 ha $^{-1}$ resulted in better management of the weeds followed by oxyfluorfen $2.5 \%+$ glyphosate $41 \%$ SC @ 3.1251 ha $^{-1}$. The weed control efficiency based on weed dry weight over control at 45 and 60 DAA resulted in better efficacy of these herbicide doses .It observed that plots treated with oxyfluorfen $2.5 \%+$ glyphosate $41 \%$ SC @ $3.6251 \mathrm{ha}^{-1}$ and $3.125 \mathrm{l} \mathrm{ha}^{-1}$ provided significantly superior management of the weeds in grape vineyard compared to other treatments. Among the herbicides, significantly higher grape yield $\left(21.83 \mathrm{t} / \mathrm{ha}\right.$ ) was recorded with application of oxyfluorfen $2.5 \%$ + glyphosate $41 \%$ SC @ $3.6251 \mathrm{ha}^{-1}$ which was on par with oxyfluorfen $2.5 \%+$ glyphosate $41 \%$ SC @ 3.1251 ha $^{-1}$ (21.68 t/ha). Higher monetary benefit cost ratio ( 9.60) was recorded with oxyfluorfen $2.5 \%+$ glyphosate $41 \%$ SC @ $3.1251 \mathrm{ha}^{-1}$ followed by oxyfluorfen $2.5 \%+$ glyphosate $41 \%$ SC @ $3.6251 \mathrm{ha}^{-1}$ (9.32). The hand weeding treatment showed lower benefit cost ratio (5.76). Other treatments were economically not beneficial to control weeds in grape crop. No symptoms of phytotoxicity were observed at $1,3,5,7$ and 10 days after the application with oxyfluorfen $2.5 \%$ + glyphosate $41 \%$ SC @ 3.125 and 6.251 $\mathrm{ha}^{-1}$.
\end{abstract}

\section{Introduction}

Grape (Vitis vinifera L.) is one of the most important fruit crops of temperate zone, which has acclimatized to sub-tropical and tropical agro climatic conditions prevailing in the Indian subcontinent. In India, grapes are grown under different soil and cultural conditions. Grapevines and weeds compete for water, nutrients, and light. The primary goal of weed management is to optimize yield by minimizing weed competition. Weeds reduce yields by competing with crops. Weeds reduce harvest efficiency, as well. timely weed removal, wise use of herbicides, and prevention of weed seed production are an integral part of a good weed management system.

Weed flora varies according to the climate and physio-chemical properties of the soil. Irrespective of the agro climatic conditions, 
Parthenium histerophorus, Cynodon dactylon Cyperus rotundus are the common weeds in the Indian vineyards although as many as 378 species of weeds have been reported to infest the cultivated lands in Karnataka (Krishna Sastry et al., 1980). Weed menace has been considered as one of the major constraints in grape production. Timely weeding reduces the crop-weed competition, resulting higher crop yields (Stevenson et al., 1986). Hand weeding, though an efficient method, is laborious, costly, time consuming and unsuitable for large grape vineyards (Bajwa et al., 1989).

This necessiates the use of herbicides for weed management in developing countries like India. In the past, majority of workers have tried either pre-emergence or postemergence application of herbicides for the control of weeds in the grape vineyard. No single herbicide either as pre-emergence or post-emergence can offer a long lasting control of weeds in vineyards since grape vines are irrigated and the soil moisture is maintained throughout the year, which helps the weeds to grow almost throughout the year. Keeping all these aspects in mind, the present investigation was undertaken to study the bioefficacy and phytotoxicity of Oxyfluorfen $2.5 \%$ + Glyphosate $41 \%$ SC (Ready mix) against Weed Complex in Grape.

\section{Materials and Methods}

A field experiment was conducted on grape (Vitis vinifera L.cv. Thompson seedless) at the vineyard of the farmer's field (Location latitude $16.52 \mathrm{~N}$ and longitude $75.69 \mathrm{E}$ ) of Vijayapura district coming under the jursdiction of University of Agricultural Sciences Dharwad, Karnataka, India. after October pruning of 2014-15 and 2015-16. The trial was laid out in randomized complete block design, having eight treatments with three replications, to test the effect of various treatments for managing weeds in the grape vineyards under tropical conditions of Vijayapura, Karnataka. The vines selected were spaced at $2.4 \mathrm{~m}$ between rows and $1.2 \mathrm{~m}$ within rows. The plot size was $9.6 \mathrm{~m} \times 6.0 \mathrm{~m}$ accommodating 20 vines in each treatment. Irrigation and fertilizer requirements were followed as per recommended package. All the herbicides were applied at 3 to 4 leaf stage of weed in vineyard using spray volume 5001 $\mathrm{ha}^{-1}$ with the help of knapsack sprayer fitted with a flood jet nozzle WFN 0.040. Weeds were counted by taking a quadrant of $1 \mathrm{~m} \mathrm{x}$ $1 \mathrm{~m}$ placed at random inside the each treated plot. The total number of dicot and monocot weeds present in the quadrant frame was counted at 45 and 60 days after herbicide treatment. The sum of all weeds was recorded as total weed count per square meter. For dry weight, the above ground portion of the weeds in the quadrant was collected from each plot at 45 and 60 days after treatment.

The weed samples were air dried and later oven dried to constant weight at $60 \mathrm{oC}$ and dry weight was recorded. Based on weed dry weight weed control efficiency was calculated. Grape yield per hectare was calculated based on bunch weight at the time of crop harvest. The yield of ten vines in each treatment was recorded and average yield per hectare was calculated and expressed in tonnes. Benefit cost ratio using cost of inputs used was also calculated. All weed parameters studied showed high degree of variation. Therefore, data was subjected to square-root transformation as suggested by Gomez and Gomez (1984) to make the analysis of variance valid. The dominant weed flora includes grasses - Cynondon dactylon, Setaria verticiliata, Digitaria sanguinalis. Broadleaf Parthenium hysterophorus, Euphorbia hirta, Portulaca oleracea, Tridax procumbens, Amaranthus sp, Phyllanthus maderaspatensis, Cardiospermum helicacabum Cleome viscose and sedges - Cyperus rotundus. The 
observation on phytotoxicity of grape vine was recorded at $1,3,5,7$ and 10 days after application of herbicide on the basis of rating scale (PRS). The parameters on phytotoxicity were recorded considering vain clearing, chlorosis, necrosis, wilting, scorching, hyponasty and epinasty. The observation on the level of phytotoxicity through visual assessment of crop response was rated on the scale of $0-10$. Rating " 0 " indicates no visible injury and "10" indicates the complete destruction of the crop. Hand weeding treatment was also included. The treatments details are as given below (Table 1).

\section{Results and Discussion}

\section{Effect of herbicides on weed density}

Herbicide treatments in grape vineyard significantly reduced weed population. Lowest weed density $\left(3.0 \mathrm{~m}^{2}\right)$ was observed after 45 days of application of Oxyfluorfen $2.5 \%$ + Glyphosate 41\% SC @ $3.6251 \mathrm{ha}^{-1}$ followed by application of Oxyfluorfen $2.5 \%$ + Glyphosate 41\% SC @ $3.1251 \mathrm{ha}^{-1}$ ( $3.2 \mathrm{~m}^{-}$

$\left.{ }^{2}\right)$. However, after 60 days of spraying of herbicides, highest weed control was observed with lowest weed density $\left(3.6 \mathrm{~m}^{-}{ }^{2}\right)$ in Oxyfluorfen $2.5 \%$ + Glyphosate $41 \%$ SC (a) $3.6251 \mathrm{ha}^{-1}$ treated plot compared to rest of the herbicides followed by the same herbicide applied@3.125 1 ha ${ }^{-1}$. This might be due to persistence of glyphosate for a longer period.
Similar results were observed by Gaziev and Voevodin (1985) who showed that glyphosate persisted in soil for three to five months under rainfed conditions and three months under irrigated conditions (Table 2 and 3). After 60 days of spraying highest weed density was recorded in weedy check treatment which was in contrast with the findings of Rekha et al., (2002) and Hussain et al., (2008). The results are in conformation with the earlier works by Hebbethwaite and Schepens (1986), Bajwa (1990) and Bajwa et al., (1992), who also reported glyphosate as very effective for controlling both mono and dicot weeds in grapes.

\section{Effect of herbicides on dry weight of weeds and weed control efficiency}

Application of Oxyfluorfen $2.5 \%+$ Glyphosate $41 \%$ SC (RM) with other herbicides treatments in 'Thompson seedless' grape vineyard significantly reduced the dry weight of all types of weeds both at 45 and 60 days compared to weedy check. Oxyfluorfen $2.5 \%$ + Glyphosate 41\% SC @ $3.625 \mathrm{ha}^{-1}$ or $3.1251 \mathrm{ha}^{-1}$ showed its superiority by recording lower dry weight of weeds compared to other post emergent herbicides like glyphosate $41 \%$ SL, diuron $\% 80 \mathrm{WP}$ and oxyfluorfen $23.5 \%$ EC (Table 3 and 4 ). Similar results were obtained by Bajwa et al., (1993) and Muniyappa and Prathibha (1993).

Table.1 Treatment detail

\begin{tabular}{|c|c|c|c|}
\hline \multirow[t]{2}{*}{ T. No. } & \multirow[t]{2}{*}{ Treatment } & \multicolumn{2}{|c|}{ Dose $_{h a}{ }^{-1}$} \\
\hline & & a. i. (g) & Formulation (l or kg ha ${ }^{-1}$ ) \\
\hline 1. & Oxyfluorfen $2.5 \%+$ Glyphosate $41 \%$ SC* & $65.625+1076.25$ & 2.625 \\
\hline 2. & Oxyfluorfen $2.5 \%+$ Glyphosate $41 \%$ SC* & $78.125+1281.25$ & 3.125 \\
\hline 3. & Oxyfluorfen $2.5 \%+$ Glyphosate $41 \%$ SC* & $90.625+1486.25$ & 3.625 \\
\hline 4. & Oxyfluorfen $23.5 \%$ EC & 250 & 1.00 \\
\hline 5. & Glyphosate $41 \%$ SL & 1230 & 3.00 \\
\hline 6. & Diuron $80 \%$ WP & 1600 & 2.00 \\
\hline 7. & Weedy check & - & - \\
\hline 8. & Weed free check & - & - \\
\hline
\end{tabular}


Table.2 Effect of Oxyfluorfen $2.5 \%+$ Glyphosate $41 \%$ SC and other herbicides on weed density $\left(\mathrm{m}^{-2}\right)$ at 45 DAA in grapes (Pooled data of two years)

\begin{tabular}{|c|c|c|c|c|c|c|c|c|c|c|c|c|}
\hline $\begin{array}{l}\text { Sl. } \\
\text { No. }\end{array}$ & Treatment & $\begin{array}{c}\text { Dose } \\
\text { l or } \\
\mathrm{kg} / \mathrm{ha}\end{array}$ & $\begin{array}{l}\text { Cynodon } \\
\text { dactylon }\end{array}$ & $\begin{array}{c}\text { Setaria } \\
\text { verticiliata }\end{array}$ & $\begin{array}{c}\text { Parthenium } \\
\text { hysterophorus }\end{array}$ & $\begin{array}{l}\text { Euphorbia } \\
\text { hirta }\end{array}$ & $\begin{array}{c}\text { Portulaca } \\
\text { oleracea }\end{array}$ & $\begin{array}{c}\text { Tridax } \\
\text { procumbens }\end{array}$ & $\begin{array}{l}\text { Amaranthus } \\
\text { spp. }\end{array}$ & $\begin{array}{l}\text { Cyperus } \\
\text { rotundus }\end{array}$ & $\begin{array}{l}\text { Other } \\
\text { weeds }\end{array}$ & Total \\
\hline \multirow[t]{2}{*}{1.} & \multirow{2}{*}{$\begin{array}{l}\text { Oxyfluorfen } 2.5 \% \\
+ \text { Glyphosate } \\
41 \% \text { SC directed } \\
\text { spray as PoE }\end{array}$} & \multirow[t]{2}{*}{2.625} & 3.56 & 2.89 & 2.11 & 2.67 & 1.22 & 1.44 & 1.67 & 6.22 & 1.00 & 22.8 \\
\hline & & & $(2.01)^{*}$ & (1.84) & (1.62) & (1.77) & $(1.31)$ & (1.39) & $(1.47)$ & $(2.59)$ & $(1.22)$ & (4.8) \\
\hline \multirow[t]{2}{*}{2.} & \multirow{2}{*}{$\begin{array}{l}\text { Oxyfluorfen } 2.5 \% \\
+ \text { Glyphosate } \\
41 \% \text { SC }\end{array}$} & \multirow[t]{2}{*}{3.125} & 1.89 & 1.56 & 1.22 & 1.56 & 0.00 & 0.00 & 0.22 & 3.00 & 0.22 & 9.7 \\
\hline & & & (1.54) & $(1.43)$ & $(1.31)$ & (1.43) & $(0.71)$ & $(0.71)$ & $(0.83)$ & $(1.87)$ & $(0.85)$ & (3.2) \\
\hline \multirow[t]{2}{*}{3.} & \multirow{2}{*}{$\begin{array}{l}\text { Oxyfluorfen } 2.5 \% \\
+ \text { Glyphosate } \\
41 \% \text { SC }\end{array}$} & \multirow[t]{2}{*}{3.625} & 1.67 & 1.56 & 1.00 & 1.33 & 0.00 & 0.00 & 0.00 & 2.56 & 0.11 & 8.2 \\
\hline & & & (1.47) & (1.43) & $(1.22)$ & (1.34) & $(0.71)$ & $(0.71)$ & $(0.71)$ & (1.69) & $(0.78)$ & (3.0) \\
\hline \multirow[t]{2}{*}{4.} & \multirow{2}{*}{$\begin{array}{l}\text { Oxyfluorfen } \\
23.5 \% \text { EC } \\
\text { directed spray as } \\
\text { PoE }\end{array}$} & \multirow[t]{2}{*}{1.000} & 7.56 & 6.67 & 1.89 & 2.44 & 0.78 & 0.78 & 1.11 & 4.89 & 0.67 & 26.8 \\
\hline & & & (2.83) & $(2.67)$ & (1.54) & (1.71) & (1.10) & (1.13) & $(1.25)$ & $(2.32)$ & $(1.05)$ & (5.2) \\
\hline \multirow[t]{2}{*}{5.} & \multirow{2}{*}{$\begin{array}{l}\text { Glyphosate } 41 \% \\
\text { SL directed spray } \\
\text { as PoE }\end{array}$} & \multirow[t]{2}{*}{3.000} & 2.33 & 1.89 & 1.67 & 2.11 & 0.67 & 0.56 & 0.78 & 4.56 & 0.44 & 15.0 \\
\hline & & & (1.68) & (1.54) & (1.47) & (1.62) & (1.08) & $(1.02)$ & (1.13) & $(2.24)$ & $(0.97)$ & (3.9) \\
\hline \multirow[t]{2}{*}{6.} & \multirow{2}{*}{$\begin{array}{l}\text { Diuron } 80 \% \text { WP } \\
\text { directed spray as } \\
\text { PoE }\end{array}$} & \multirow{2}{*}{$\begin{array}{c}2 \\
.000\end{array}$} & 2.56 & 2.11 & 1.78 & 2.33 & 0.78 & 0.56 & 1.00 & 4.78 & 0.56 & 16.5 \\
\hline & & & $(1.75)$ & $(1.62)$ & $(1.51)$ & $(1.68)$ & $(1.13)$ & $(1.02)$ & $(1.22)$ & $(2.29)$ & $(1.02)$ & (4.1) \\
\hline \multirow[t]{2}{*}{7.} & \multirow[t]{2}{*}{ Weedy check } & \multirow[t]{2}{*}{ - } & 11.33 & 8.67 & 6.44 & 7.89 & 4.44 & 5.00 & 5.78 & 16.44 & 3.78 & 69.8 \\
\hline & & & $(3.44)$ & $(3.03)$ & $(2.62)$ & $(2.89)$ & $(2.22)$ & $(2.34)$ & $(2.50)$ & (4.11) & $(2.07)$ & (8.4) \\
\hline \multirow[t]{4}{*}{8.} & \multirow[t]{2}{*}{ Weed free check } & & 1.56 & 1.33 & 0.89 & 1.11 & 0.00 & 0.00 & 0.00 & 2.44 & 0.11 & 7.4 \\
\hline & & & $(1.43)$ & $(1.35)$ & (1.18) & $(1.27)$ & $(0.71)$ & $(0.71)$ & $(0.71)$ & (1.71) & $(0.78)$ & (2.8) \\
\hline & SEm \pm & & 0.04 & 0.03 & 0.05 & 0.05 & 0.07 & 0.05 & 0.07 & 0.09 & 0.04 & 0.27 \\
\hline & $\mathrm{CD}(\mathrm{P}=0.05)$ & & 0.11 & 0.10 & 0.15 & 0.16 & 0.23 & 0.14 & 0.21 & 0.28 & 0.11 & 0.82 \\
\hline
\end{tabular}

Other weeds - Cleome viscosa, Digitaria sanguinalis, Phyllanthus madraspatensis and Cardiospermum helicacabum;

*Data in the parentheses indicate the transformed values $\sqrt{x}+0.5$, PoE: Post emergence 
Table.3 Effect of Oxyfluorfen $2.5 \%$ + Glyphosate $41 \%$ SC and other herbicides on weed density $\left(\mathrm{m}^{-2}\right)$ at 60 DAA in grapes (Pooled data of two years)

\begin{tabular}{|c|c|c|c|c|c|c|c|c|c|c|c|c|}
\hline $\begin{array}{l}\text { SI. } \\
\text { No. }\end{array}$ & Treatment & $\begin{array}{c}\text { Dose } \\
\text { l or } \\
\mathrm{kg} / \mathrm{ha}\end{array}$ & $\begin{array}{l}\text { Cynodon } \\
\text { dactylon }\end{array}$ & $\begin{array}{c}\text { Setaria } \\
\text { verticiliata }\end{array}$ & $\begin{array}{c}\text { Parthenium } \\
\text { hysterophorus }\end{array}$ & $\begin{array}{c}\text { Euphorbia } \\
\quad \text { hirta }\end{array}$ & $\begin{array}{l}\text { Portulaca } \\
\text { oleracea }\end{array}$ & $\begin{array}{c}\text { Tridax } \\
\text { procumbens }\end{array}$ & $\begin{array}{c}\text { Amaranthus } \\
\text { spp. }\end{array}$ & $\begin{array}{c}\text { Cyperus } \\
\text { rotundus }\end{array}$ & $\begin{array}{l}\text { Other } \\
\text { weeds }\end{array}$ & Total \\
\hline \multirow[t]{2}{*}{1.} & \multirow{2}{*}{$\begin{array}{l}\text { Oxyfluorfen } 2.5 \% \\
+ \text { Glyphosate } 41 \% \\
\text { SC directed spray } \\
\text { as PoE }\end{array}$} & \multirow[t]{2}{*}{2.625} & 3.78 & 3.11 & 2.67 & 3.00 & 1.56 & 1.67 & 2.00 & 6.56 & 1.22 & 25.6 \\
\hline & & & $(2.07)^{*}$ & (1.90) & (1.78) & (1.87) & (1.43) & (1.47) & (1.58) & (2.65) & $(1.31)$ & (5.1) \\
\hline \multirow[t]{2}{*}{2.} & \multirow{2}{*}{$\begin{array}{l}\text { Oxyfluorfen } 2.5 \% \\
+ \text { Glyphosate } 41 \% \\
\text { SC }\end{array}$} & \multirow[t]{2}{*}{3.125} & 1.89 & 2.00 & 1.56 & 1.56 & 0.56 & 0.78 & 0.67 & 3.11 & 0.44 & 12.6 \\
\hline & & & (1.54) & (1.58) & (1.41) & $(1.43)$ & $(1.02)$ & (1.13) & $(1.08)$ & $(1.90)$ & $(0.97)$ & (3.6) \\
\hline \multirow[t]{2}{*}{3.} & \multirow{2}{*}{$\begin{array}{l}\text { Oxyfluorfen } 2.5 \% \\
+ \text { Glyphosate } 41 \% \\
\text { SC }\end{array}$} & \multirow[t]{2}{*}{3.625} & 1.78 & 1.78 & 1.33 & 1.44 & 0.33 & 0.56 & 0.67 & 3.00 & 0.33 & 11.2 \\
\hline & & & $(1.51)$ & $(1.50)$ & $(1.35)$ & (1.39) & $(0.91)$ & $(1.00)$ & (1.08) & (1.79) & $(0.91)$ & (3.4) \\
\hline \multirow[t]{2}{*}{4.} & \multirow{2}{*}{$\begin{array}{l}\text { Oxyfluorfen } 23.5 \% \\
\text { EC directed spray } \\
\text { as PoE }\end{array}$} & \multirow[t]{2}{*}{1.000} & 7.78 & 6.89 & 2.44 & 2.56 & 1.00 & 1.33 & 1.67 & 5.22 & 0.78 & 29.7 \\
\hline & & & $(2.87)$ & $(2.71)$ & (1.71) & (1.74) & (1.22) & (1.35) & (1.47) & (2.39) & (1.09) & (5.5) \\
\hline \multirow[t]{2}{*}{5.} & \multirow{2}{*}{$\begin{array}{l}\text { Glyphosate } 41 \% \text { SL } \\
\text { directed spray as } \\
\text { PoE }\end{array}$} & \multirow[t]{2}{*}{3.000} & 2.44 & 2.67 & 2.11 & 2.22 & 0.89 & 1.22 & 1.33 & 5.00 & 0.67 & 18.6 \\
\hline & & & (1.71) & (1.78) & $(1.62)$ & $(1.65)$ & (1.18) & $(1.31)$ & $(1.35)$ & $(2.34)$ & $(1.08)$ & (4.4) \\
\hline \multirow[t]{2}{*}{6.} & \multirow{2}{*}{$\begin{array}{l}\text { Diuron } 80 \% \mathrm{WP} \\
\text { directed spray as } \\
\text { PoE }\end{array}$} & \multirow{2}{*}{$\begin{array}{c}2 \\
.000\end{array}$} & 2.67 & 2.78 & 2.33 & 2.33 & 1.00 & 1.22 & 1.44 & 5.00 & 0.78 & 19.6 \\
\hline & & & (1.78) & $(1.81)$ & (1.68) & (1.68) & $(1.22)$ & (1.31) & (1.39) & (2.34) & (1.13) & (4.5) \\
\hline \multirow[t]{2}{*}{7.} & \multirow[t]{2}{*}{ Weedy check } & \multirow[t]{2}{*}{ - } & 13.67 & 10.00 & 7.56 & 8.44 & 5.22 & 5.89 & 6.44 & 18.22 & 4.00 & 79.4 \\
\hline & & & $(3.76)$ & $(3.23)$ & $(2.83)$ & $(2.98)$ & (2.39) & $(2.52)$ & $(2.63)$ & $(4.32)$ & $(2.12)$ & (8.9) \\
\hline \multirow[t]{4}{*}{8.} & \multirow[t]{2}{*}{ Weed free check } & \multirow[t]{2}{*}{ - } & 1.78 & 1.56 & 1.11 & 1.33 & 0.33 & 0.44 & 0.44 & 2.78 & 0.33 & 10.1 \\
\hline & & & $(1.51)$ & $(1.43)$ & $(1.27)$ & $(1.35)$ & $(0.91)$ & $(0.97)$ & $(0.97)$ & $(1.81)$ & $(0.91)$ & (3.3) \\
\hline & SEm \pm & & 0.05 & 0.05 & 0.06 & 0.04 & 0.05 & 0.05 & 0.04 & 0.12 & 0.03 & 0.11 \\
\hline & $\mathrm{CD}(\mathrm{P}=\mathbf{0 . 0 5})$ & & 0.15 & 0.16 & 0.19 & 0.13 & 0.14 & 0.16 & 0.13 & 0.37 & 0.09 & 0.32 \\
\hline
\end{tabular}

Other weeds - Cleome viscosa, Digitaria sanguinalis, Phyllanthus madraspatensis and Cardiospermum helicacabum

*Data in the parentheses indicate the transformed values $\sqrt{x}+0.5$, PoE: Post emergence 
Table.4 Effect of Oxyfluorfen 2.5\% + Glyphosate 41\% SC and other herbicides on weed biomass $\left(\mathrm{g} \mathrm{m}^{-2}\right)$ at 45 DAA in grapes (Pooled data of two years)

\begin{tabular}{|c|c|c|c|c|c|c|c|c|c|c|c|c|}
\hline $\begin{array}{l}\text { Sl. } \\
\text { No. }\end{array}$ & Treatment & $\begin{array}{l}\text { Dose } \\
\text { l or } \\
\text { kg/ha }\end{array}$ & $\begin{array}{l}\text { Cynodon } \\
\text { dactylon }\end{array}$ & $\begin{array}{c}\text { Setaria } \\
\text { verticiliata }\end{array}$ & $\begin{array}{c}\text { Parthenium } \\
\text { hysterophorus }\end{array}$ & $\begin{array}{c}\text { Euphorbia } \\
\text { hirta }\end{array}$ & $\begin{array}{c}\text { Portulaca } \\
\text { oleracea }\end{array}$ & $\begin{array}{c}\text { Tridax } \\
\text { procumbens }\end{array}$ & $\begin{array}{l}\text { Amaranthus } \\
\text { spp. }\end{array}$ & $\begin{array}{l}\text { Cyperus } \\
\text { rotundus }\end{array}$ & $\begin{array}{l}\text { Other } \\
\text { Weeds }\end{array}$ & Total \\
\hline \multirow[t]{2}{*}{1.} & \multirow{2}{*}{$\begin{array}{l}\text { Oxyfluorfen } 2.5 \%+ \\
\text { Glyphosate } 41 \% \text { SC } \\
\text { directed spray as } \\
\text { PoE }\end{array}$} & \multirow[t]{2}{*}{2.625} & 2.73 & 1.86 & 2.18 & 2.86 & 1.36 & 1.86 & 1.66 & 3.00 & 1.42 & 18.9 \\
\hline & & & $(1.80)^{*}$ & (1.53) & (1.63) & (1.83) & (1.36) & (1.53) & $(1.47)$ & (1.87) & (1.38) & (4.4) \\
\hline \multirow[t]{2}{*}{2.} & \multirow{2}{*}{$\begin{array}{l}\text { Oxyfluorfen } 2.5 \%+ \\
\text { Glyphosate } 41 \% \text { SC }\end{array}$} & \multirow[t]{2}{*}{3.125} & 1.14 & 0.73 & 0.88 & 1.06 & 0.00 & 0.00 & 0.46 & 1.38 & 0.34 & 6.0 \\
\hline & & & $(1.28)$ & (1.11) & (1.17) & $(1.25)$ & $(0.71)$ & $(0.71)$ & $(0.98)$ & $(1.37)$ & $(0.92)$ & (2.5) \\
\hline \multirow[t]{2}{*}{3.} & \multirow{2}{*}{$\begin{array}{l}\text { Oxyfluorfen } 2.5 \%+ \\
\text { Glyphosate } 41 \% \text { SC }\end{array}$} & \multirow[t]{2}{*}{3.625} & 1.02 & 0.69 & 0.82 & 1.00 & 0.00 & 0.00 & 0.00 & 1.25 & 0.26 & 5.0 \\
\hline & & & (1.23) & (1.09) & (1.15) & $(1.22)$ & $(0.71)$ & $(0.71)$ & $(0.71)$ & $(1.32)$ & $(0.87)$ & (2.4) \\
\hline \multirow[t]{2}{*}{4.} & \multirow{2}{*}{$\begin{array}{l}\text { Oxyfluorfen } 23.5 \% \\
\text { EC directed spray } \\
\text { as PoE }\end{array}$} & \multirow[t]{2}{*}{1.000} & 3.86 & 2.73 & 1.74 & 1.83 & 1.00 & 1.42 & 1.26 & 2.11 & 0.98 & 16.9 \\
\hline & & & $(2.08)$ & $(1.79)$ & (1.49) & $(1.51)$ & $(1.20)$ & $(1.38)$ & $(1.33)$ & (1.59) & $(1.22)$ & $(4.2)$ \\
\hline \multirow[t]{2}{*}{5.} & \multirow{2}{*}{$\begin{array}{l}\text { Glyphosate } 41 \% \text { SL } \\
\text { directed spray as } \\
\text { PoE }\end{array}$} & \multirow[t]{2}{*}{3.000} & 1.85 & 1.30 & 1.63 & 1.73 & 0.96 & 1.31 & 1.18 & 2.02 & 0.92 & 12.9 \\
\hline & & & $(1.52)$ & (1.34) & $(1.46)$ & (1.49) & $(1.21)$ & (1.34) & $(1.28)$ & (1.59) & $(1.15)$ & (3.7) \\
\hline \multirow[t]{2}{*}{6.} & \multirow{2}{*}{$\begin{array}{l}\text { Diuron } 80 \% \text { WP } \\
\text { directed spray as } \\
\text { PoE }\end{array}$} & \multirow[t]{2}{*}{2.000} & 1.93 & 1.40 & 1.69 & 1.82 & 0.97 & 1.36 & 1.23 & 2.10 & 0.95 & 13.5 \\
\hline & & & $(1.56)$ & $(1.38)$ & $(1.48)$ & $(1.52)$ & $(1.21)$ & (1.36) & (1.31) & (1.61) & $(1.20)$ & (3.7) \\
\hline \multirow[t]{2}{*}{7.} & \multirow[t]{2}{*}{ Weedy check } & \multirow[t]{2}{*}{-} & 9.74 & 7.02 & 8.63 & 9.70 & 5.06 & 6.80 & 6.24 & 10.69 & 4.88 & 68.8 \\
\hline & & & (3.19) & (2.74) & $(3.00)$ & (3.19) & $(2.35)$ & $(2.70)$ & $(2.57)$ & $(3.34)$ & $(2.31)$ & (8.3) \\
\hline \multirow[t]{4}{*}{8.} & \multirow[t]{2}{*}{ Weed free check } & & 0.86 & 0.58 & 0.63 & 0.90 & 0.00 & 0.00 & 0.00 & 1.02 & 0.18 & 4.2 \\
\hline & & & (1.17) & (1.04) & (1.06) & (1.18) & $(0.71)$ & $(0.71)$ & $(0.71)$ & $(1.23)$ & $(0.82)$ & (2.2) \\
\hline & SEm \pm & & 0.05 & 0.03 & 0.08 & 0.05 & 0.06 & 0.04 & 0.09 & 0.06 & 0.07 & 0.04 \\
\hline & $\mathrm{CD}(\mathrm{P}=\mathbf{0 . 0 5})$ & & 0.14 & 0.10 & 0.23 & 0.15 & 0.18 & 0.13 & 0.27 & 0.18 & 0.20 & 0.12 \\
\hline
\end{tabular}

Other weeds - Cleome viscosa, Digitaria sanguinalis, Phyllanthus madraspatensis and Cardiospermum helicacabum

*Data in the parentheses indicate the transformed values $\sqrt{x}+0.5$, PoE: Post emergence 
Table.5 Effect of Oxyfluorfen 2.5\% + Glyphosate 41\% SC and other herbicides on weed biomass $\left(\mathrm{g} \mathrm{m}^{-2}\right)$ at 60DAA in grapes (Pooled data of two years)

\begin{tabular}{|c|c|c|c|c|c|c|c|c|c|c|c|c|}
\hline $\begin{array}{l}\text { Sl. } \\
\text { No. }\end{array}$ & Treatment & $\begin{array}{c}\text { Dose } \\
\text { l or } \\
\text { kg/ha }\end{array}$ & $\begin{array}{l}\text { Cynodon } \\
\text { dactylon }\end{array}$ & $\begin{array}{c}\text { Setaria } \\
\text { verticiliata }\end{array}$ & $\begin{array}{c}\text { Parthenium } \\
\text { hysterophorus }\end{array}$ & $\begin{array}{c}\text { Euphorbia } \\
\quad \text { hirta }\end{array}$ & $\begin{array}{c}\text { Portulaca } \\
\text { oleracea }\end{array}$ & $\begin{array}{c}\text { Tridax } \\
\text { procumbens }\end{array}$ & $\begin{array}{c}\text { Amaranthus } \\
\text { spp. }\end{array}$ & $\begin{array}{l}\text { Cyperus } \\
\text { rotundus }\end{array}$ & $\begin{array}{l}\text { Other } \\
\text { Weeds }\end{array}$ & Total \\
\hline \multirow[t]{2}{*}{1.} & \multirow{2}{*}{$\begin{array}{l}\text { Oxyfluorfen } 2.5 \%+ \\
\text { Glyphosate } 41 \% \text { SC } \\
\text { directed spray as } \\
\text { PoE }\end{array}$} & \multirow[t]{2}{*}{2.625} & 4.01 & 2.75 & 3.36 & 4.16 & 2.00 & 2.62 & 2.21 & 4.32 & 1.73 & 27.2 \\
\hline & & & $(2.12)^{*}$ & $(1.80)$ & (1.96) & $(2.15)$ & $(1.58)$ & $(1.76)$ & (1.64) & (2.19) & $(1.49)$ & (5.3) \\
\hline \multirow[t]{2}{*}{2.} & \multirow{2}{*}{$\begin{array}{l}\text { Oxyfluorfen } 2.5 \%+ \\
\text { Glyphosate } 41 \% \text { SC }\end{array}$} & \multirow[t]{2}{*}{3.125} & 1.51 & 1.03 & 1.36 & 1.58 & 0.77 & 0.95 & 0.82 & 2.03 & 0.54 & 10.6 \\
\hline & & & $(1.42)$ & $(1.24)$ & $(1.36)$ & $(1.44)$ & (1.13) & $(1.20)$ & $(1.15)$ & $(1.59)$ & $(1.02)$ & (3.3) \\
\hline \multirow[t]{2}{*}{3.} & \multirow{2}{*}{$\begin{array}{l}\text { Oxyfluorfen } 2.5 \%+ \\
\text { Glyphosate } 41 \% \text { SC }\end{array}$} & \multirow[t]{2}{*}{3.625} & 1.46 & 1.00 & 1.28 & 1.50 & 0.73 & 0.88 & 0.79 & 1.97 & 0.41 & 10.0 \\
\hline & & & $(1.40)$ & $(1.22)$ & (1.33) & $(1.41)$ & (1.11) & $(1.17)$ & (1.13) & $(1.57)$ & $(0.95)$ & (3.2) \\
\hline \multirow[t]{2}{*}{4.} & \multirow{2}{*}{$\begin{array}{l}\text { Oxyfluorfen } 23.5 \% \\
\text { EC directed spray } \\
\text { as PoE }\end{array}$} & \multirow[t]{2}{*}{1.000} & 5.86 & 3.80 & 2.46 & 2.82 & 1.44 & 1.73 & 1.58 & 3.00 & 1.28 & 24.0 \\
\hline & & & $(2.52)$ & (2.07) & $(1.72)$ & $(1.82)$ & (1.39) & (1.49) & $(1.44)$ & $(1.87)$ & $(1.33)$ & (4.9) \\
\hline \multirow[t]{2}{*}{5.} & \multirow{2}{*}{$\begin{array}{l}\text { Glyphosate } 41 \% \text { SL } \\
\text { directed spray as } \\
\text { PoE }\end{array}$} & \multirow[t]{2}{*}{3.000} & 2.62 & 1.75 & 2.33 & 2.70 & 1.31 & 1.62 & 1.49 & 2.87 & 1.16 & 17.9 \\
\hline & & & $(1.76)$ & $(1.50)$ & $(1.68)$ & (1.79) & $(1.34)$ & $(1.45)$ & $(1.41)$ & $(1.83)$ & $(1.29)$ & (4.3) \\
\hline \multirow[t]{2}{*}{6.} & \multirow{2}{*}{$\begin{array}{l}\text { Diuron } 80 \% \text { WP } \\
\text { directed spray as } \\
\text { PoE }\end{array}$} & \multirow[b]{2}{*}{2.000} & 2.69 & 1.82 & 2.41 & 2.79 & 1.36 & 1.70 & 1.55 & 2.92 & 1.21 & 18.5 \\
\hline & & & $(1.78)$ & $(1.52)$ & $(1.70)$ & (1.81) & $(1.36)$ & $(1.48)$ & $(1.43)$ & $(1.85)$ & $(1.31)$ & (4.4) \\
\hline \multirow[t]{2}{*}{7.} & \multirow[t]{2}{*}{ Weedy check } & \multirow[t]{2}{*}{-} & 12.58 & 8.70 & 11.19 & 13.25 & 6.21 & 7.95 & 7.28 & 13.67 & 5.52 & 86.4 \\
\hline & & & $(3.61)$ & (3.03) & $(3.41)$ & $(3.70)$ & $(2.59)$ & $(2.90)$ & $(2.78)$ & $(3.76)$ & $(2.45)$ & (9.3) \\
\hline \multirow[t]{4}{*}{8.} & \multirow[t]{2}{*}{ Weed free check } & \multirow[t]{2}{*}{ - } & 1.25 & 0.95 & 1.16 & 1.41 & 0.63 & 0.82 & 0.73 & 1.81 & 0.30 & 9.1 \\
\hline & & & $(1.32)$ & $(1.20)$ & $(1.29)$ & $(1.38)$ & $(1.06)$ & $(1.15)$ & (1.11) & $(1.52)$ & $(0.89)$ & (3.1) \\
\hline & SEm \pm & & 0.04 & 0.03 & 0.04 & 0.04 & 0.03 & 0.03 & 0.03 & 0.04 & 0.03 & 0.12 \\
\hline & $\mathrm{CD}(\mathrm{P}=\mathbf{0 . 0 5})$ & & 0.12 & 0.10 & 0.11 & 0.12 & 0.08 & 0.10 & 0.09 & 0.12 & 0.09 & 0.36 \\
\hline
\end{tabular}

Other weeds - Cleome viscosa, Digitaria sanguinalis, Phyllanthus madraspatensis and Cardiospermum helicacabum

*Data in the parentheses indicate the transformed values $\sqrt{\mathrm{x}}+0.5$, PoE: Post emergence 
Table.6 Effect of Oxyfluorfen 2.5\% + Glyphosate 41\% SC and other herbicides on species wise Weed Control Efficiency $(\%)$ in grapes at 45 days after application. (Pooled data of two years)

\begin{tabular}{|c|c|c|c|c|c|c|c|c|c|c|c|}
\hline $\begin{array}{l}\text { Sl. } \\
\text { No. }\end{array}$ & Treatment & $\begin{array}{l}\text { Dose } \\
1 \text { or } \\
\mathrm{kg} / \mathrm{ha}\end{array}$ & $\begin{array}{l}\text { Cynodon } \\
\text { dactylon }\end{array}$ & $\begin{array}{c}\text { Setaria } \\
\text { verticiliata }\end{array}$ & $\begin{array}{l}\text { Parthenium } \\
\text { hysterophorus }\end{array}$ & $\begin{array}{l}\text { Euphorbia } \\
\text { hirta }\end{array}$ & $\begin{array}{c}\text { Portulaca } \\
\text { oleracea }\end{array}$ & $\begin{array}{c}\text { Tridax } \\
\text { procumbens }\end{array}$ & $\begin{array}{l}\text { Amaranthus } \\
\text { spp. }\end{array}$ & $\begin{array}{l}\text { Cyperus } \\
\text { rotundus }\end{array}$ & $\begin{array}{l}\text { Other } \\
\text { weeds }\end{array}$ \\
\hline 1. & $\begin{array}{l}\text { Oxyfluorfen } 2.5 \%+\text { Glyphosate } 41 \% \text { SC } \\
\text { directed spray as PoE }\end{array}$ & 2.625 & 71.97 & 73.50 & 74.74 & 70.52 & 73.12 & 72.65 & 73.40 & 71.94 & 70.90 \\
\hline 2. & Oxyfluorfen $2.5 \%$ + Glyphosate $41 \%$ SC & 3.125 & 88.30 & 89.60 & 89.80 & 89.07 & 100.00 & 100.00 & 92.63 & 87.09 & 93.03 \\
\hline 3. & Oxyfluorfen $2.5 \%$ + Glyphosate $41 \%$ SC & 3.625 & 89.53 & 90.17 & 90.50 & 89.69 & 100.00 & 100.00 & 100.00 & 88.31 & 94.67 \\
\hline 4. & $\begin{array}{l}\text { Oxyfluorfen } 23.5 \% \text { EC directed spray as } \\
\text { PoE }\end{array}$ & 1.00 & 60.37 & 61.11 & 79.84 & 81.13 & 80.24 & 79.12 & 79.81 & 80.26 & 79.92 \\
\hline 5. & $\begin{array}{l}\text { Glyphosate } 41 \% \text { SL directed spray as } \\
\text { PoE }\end{array}$ & 3.00 & 81.01 & 81.48 & 81.11 & 82.16 & 81.03 & 80.74 & 81.09 & 81.10 & 81.15 \\
\hline 6. & Diuron $80 \%$ WP directed spray as PoE & 2.00 & 80.18 & 80.06 & 80.42 & 81.24 & 80.83 & 80.00 & 80.29 & 80.36 & 80.53 \\
\hline 7. & Weedy check & - & 0.00 & 0.00 & 0.00 & 0.00 & 0.00 & 0.00 & 0.00 & 0.00 & 0.00 \\
\hline 8. & Weed free check & - & 91.17 & 91.74 & 92.70 & 90.72 & 100.00 & 100.00 & 100.00 & 90.46 & 96.31 \\
\hline
\end{tabular}

Other Weeds - Cleome viscosa, Digitaria sanguinalis, Phyllanthus madraspatensis and Cardiospermum helicacabum

Table.7 Effect of Oxyfluorfen 2.5\% + Glyphosate 41\% SC and other herbicides on species wise Weed Control Efficiency (\%) in grapes at 60 days after application. (Pooled data of two years)

\begin{tabular}{|c|c|c|c|c|c|c|c|c|c|c|c|}
\hline $\begin{array}{l}\text { Sl. } \\
\text { No. }\end{array}$ & Treatment & $\begin{array}{l}\text { Dose } \\
\text { l or } \\
\mathrm{kg} / \mathrm{ha}\end{array}$ & $\begin{array}{l}\text { Cynodon } \\
\text { dactylon }\end{array}$ & $\begin{array}{c}\text { Setaria } \\
\text { verticiliata }\end{array}$ & $\begin{array}{c}\text { Parthenium } \\
\text { hysterophorus }\end{array}$ & $\begin{array}{l}\text { Euphorbia } \\
\text { hirta }\end{array}$ & $\begin{array}{c}\text { Portulaca } \\
\text { oleracea }\end{array}$ & $\begin{array}{c}\text { Tridax } \\
\text { procumbens }\end{array}$ & $\begin{array}{l}\text { Amaranthus } \\
\text { spp. }\end{array}$ & $\begin{array}{l}\text { Cyperus } \\
\text { rotundus }\end{array}$ & $\begin{array}{l}\text { Other } \\
\text { weeds }\end{array}$ \\
\hline 1. & $\begin{array}{l}\text { Oxyfluorfen } 2.5 \%+\text { Glyphosate } 41 \% \text { SC } \\
\text { directed spray as PoE }\end{array}$ & 2.625 & 68.12 & 68.39 & 69.97 & 68.60 & 67.79 & 67.04 & 69.64 & 68.40 & 68.66 \\
\hline 2. & Oxyfluorfen $2.5 \%$ + Glyphosate $41 \%$ SC & 3.125 & 88.00 & 88.16 & 87.85 & 88.08 & 87.60 & 88.05 & 88.74 & 85.15 & 90.22 \\
\hline 3. & Oxyfluorfen $2.5 \%$ + Glyphosate $41 \%$ SC & 3.625 & 88.39 & 88.51 & 88.56 & 88.68 & 88.24 & 88.93 & 89.15 & 85.59 & 92.57 \\
\hline 4. & $\begin{array}{l}\text { Oxyfluorfen } 23.5 \% \text { EC directed spray as } \\
\text { PoE }\end{array}$ & 1.000 & 53.42 & 56.32 & 78.02 & 78.72 & 76.81 & 78.24 & 78.30 & 78.05 & 76.81 \\
\hline 5. & Glyphosate $41 \%$ SL directed spray as PoE & 3.000 & 79.17 & 79.89 & 79.18 & 79.62 & 78.90 & 79.62 & 79.53 & 79.01 & 78.99 \\
\hline 6. & Diuron $80 \%$ WP directed spray as PoE & 2.000 & 78.62 & 79.08 & 78.46 & 78.94 & 78.10 & 78.62 & 78.71 & 78.64 & 78.08 \\
\hline 7. & Weedy check & - & 0.00 & 0.00 & 0.00 & 0.00 & 0.00 & 0.00 & 0.00 & 0.00 & 0.00 \\
\hline 8. & Weed free check & - & 90.06 & 89.08 & 89.63 & 89.36 & 89.86 & 89.69 & 89.97 & 86.76 & 94.57 \\
\hline
\end{tabular}

Other Weeds - Cleome viscosa, Digitaria sanguinalis, Phyllanthus madraspatensis and Cardiospermum helicacabum 
Table.8 Grape yield as influenced by herbicide treatments

\begin{tabular}{|c|c|c|c|c|c|}
\hline \multicolumn{2}{|r|}{ Treatment } & \multirow{2}{*}{\begin{tabular}{|c|c|}
$\begin{array}{c}\text { Dose } \\
\text { l or } \mathrm{kg} / \mathrm{ha}\end{array}$ \\
2.625 \\
\end{tabular}} & \multirow{2}{*}{$\begin{array}{c}2013 \\
\text { yield(t/ha) } \\
21.25\end{array}$} & \multirow{2}{*}{$\begin{array}{c}2014 \\
\text { yield(t/ha) } \\
19.65\end{array}$} & \multirow{2}{*}{$\begin{array}{c}\begin{array}{c}\text { Pooled } \\
\text { yield(t/ha) }\end{array} \\
20.45\end{array}$} \\
\hline 1. & Oxyfluorfen $2.5 \%+$ Glyphosate $41 \%$ SC directed spray as PoE & & & & \\
\hline 2. & Oxyfluorfen $2.5 \%$ + Glyphosate $41 \%$ SC & 3.125 & 22.53 & 20.83 & 21.68 \\
\hline 3. & Oxyfluorfen $2.5 \%+$ Glyphosate $41 \%$ SC & 3.625 & 22.72 & 20.95 & 21.83 \\
\hline 4. & Oxyfluorfen $23.5 \%$ EC directed spray as PoE & 1.000 & 21.53 & 19.80 & 20.66 \\
\hline 5. & Glyphosate $41 \%$ SL directed spray as $\mathrm{PoE}$ & 3.000 & 21.00 & 20.05 & 20.52 \\
\hline 6. & Diuron $80 \%$ WP directed spray as PoE & 2.000 & 21.61 & 19.92 & 20.76 \\
\hline 7. & Weedy check & - & 20.38 & 18.85 & 19.61 \\
\hline 8. & Weed free check & - & 23.17 & 21.23 & 22.20 \\
\hline \multicolumn{3}{|c|}{ Sem \pm} & 0.24 & 0.21 & 0.20 \\
\hline \multicolumn{3}{|c|}{ CD @ $(P=0.05)$} & 0.73 & 0.63 & 0.60 \\
\hline
\end{tabular}

Table.9 Incremental benefit cost ratio of Oxyfluorfen 2.5\% + Glyphosate 41\% SC on grape (Pooled data)

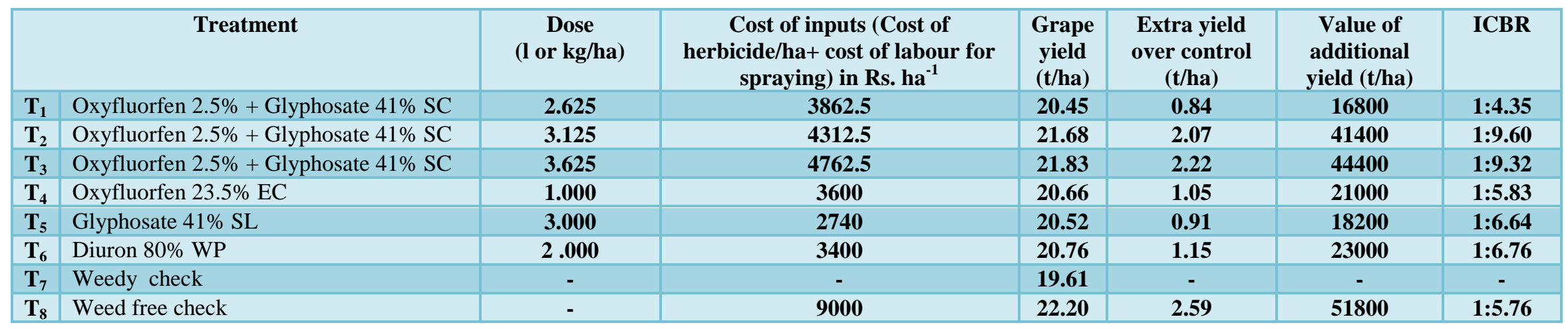

Table.10 Phytotoxicity on grape $\left(1^{\text {st }}\right.$ season and $2^{\text {nd }}$ season $)$

\begin{tabular}{|c|c|c|c|c|c|c|c|c|c|c|c|c|c|c|c|c|c|c|c|c|c|}
\hline \multirow[t]{3}{*}{ Treatment } & \multirow{3}{*}{$\begin{array}{r}\text { Dose } \\
\text { l or kg/ha }\end{array}$} & \multicolumn{5}{|c|}{ Chlorosis } & \multicolumn{5}{|c|}{ Wilting } & \multicolumn{5}{|c|}{ Hyponasity /epinasty } & \multicolumn{5}{|c|}{ Scorching } \\
\hline & & \multicolumn{20}{|c|}{ Days after herbicide application } \\
\hline & & 1 & 3 & 5 & 7 & 10 & $\mathbf{1}$ & 3 & 5 & 7 & 10 & 1 & 3 & 5 & 7 & 10 & 1 & 3 & 5 & 7 & 10 \\
\hline Oxyfluorfen $2.5 \%$ + Glyphosate $41 \%$ SC & 3.125 & 0 & 0 & 0 & 0 & 0 & 0 & 0 & 0 & 0 & 0 & 0 & 0 & 0 & 0 & 0 & 0 & 0 & 0 & 0 & 0 \\
\hline Oxyfluorfen $2.5 \%$ + Glyphosate $41 \%$ SC & 6.250 & 0 & 0 & 0 & 0 & 0 & 0 & 0 & 0 & 0 & 0 & 0 & 0 & 0 & 0 & 0 & 0 & 0 & 0 & 0 & 0 \\
\hline Control & --- & 0 & 0 & 0 & 0 & 0 & 0 & 0 & 0 & 0 & 0 & 0 & 0 & 0 & 0 & 0 & 0 & 0 & 0 & 0 & 0 \\
\hline
\end{tabular}

Scale (0-10): $0=00,1=1-10 \%, 2=11-20 \%, 3=21-30 \%, 4=31-40 \%, 5=41-50 \%, 6=51-60 \%, 7=61-70 \%, 8=71-80 \%, 9=81-90 \%, 10=91-100 \%$ 
The application of Oxyfluorfen 2.5\% + Glyphosate 41\% SC @ 3.6251 ha $^{-1}$ showed lowest dry weight $\left(2.4 \mathrm{~g} \mathrm{~m}^{2}\right)$ followed by same herbicide applied @ $3.1251 \mathrm{ha}^{-1}(2.5 \mathrm{~g}$ $\mathrm{m}^{2}$ ) after 45 days of application of herbicides. While, after 60 days, similar trend followed compared to weedy check and other herbicide treatments. These results are in conformity with the results of Mani et al., (1976), Chanana et al., (1975) and Aulakh (1999). The weed control efficiency calculated based on dry weight over weedy check at 45 and 60 DAA resulted in better efficacy of these herbicides (Table 6 and 7). It was observsd that treatments with Oxyfluorfen 2.5\% + Glyphosate 41\% SC @ $3.6251 \mathrm{ha}^{-1}$ and $3.125 \mathrm{l} \mathrm{ha}$ provided significantly superior control of the weeds in grape vineyard compared to other treatments.

\section{Effect of herbicides on grape yield and incremental benefit cost ratio}

The data on yield showed significant effect on grape yield (Table 8). Maximum yield (21.83 $\mathrm{t} \mathrm{ha}^{-1}$ ) was produced with Oxyfluorfen $2.5 \%+$ Glyphosate 41\% SC @ 3.6251 ha $^{-1}$ weedicide treatment followed by Oxyfluorfen 2.5\% + Glyphosate 41\% SC @ 3.1251 ha $^{-1}$ treatment. Increase in yield in herbicidel applied treatments and weed free check was due to increase in yield components resulting from control of weeds and lack of moisture and nutrients. Increase yield by the application of herbicides has been reported by Bajwa et al., (1993) and Bajwa et al., (1997).

Higher benefit cost ratio (Table 9) was recorded with Oxyfluorfen $2.5 \%+$ Glyphosate 41\% SC @ $3.1251 \mathrm{ha}^{-1}$ (9.60) closely followed by Oxyfluorfen 2.5\% + Glyphosate 41\% SC @ 3.6251 ha $^{-1}$ (9.32). The weed free check treatment showed lower benefit cost ratio (5.76). Other treatments were still lower economically to control weeds in grape crop.

\section{Phytotoxicity}

Symptoms of phytotoxicity on grape vine are presented in Table 10. No phytotoxic signs or symptoms viz., leaf tip/surface injury, wilting, vein clearing, necrosis, epinasty and hyponasty were observed at 1, 3, 5, 7 and 10 days after the application of Oxyfluorfen $2.5 \%$ + Glyphosate 41\% SC @ 3.125 and $6.25 \mathrm{l} \mathrm{ha}^{-1}$.

It can be concluded that highest WCE at 45 $(\%)$ and 60 DAA (\%) during was found with Oxyfluorfen $2.5 \%$ + Glyphosate $41 \%$ SC @ $3.6251 \mathrm{ha}^{-1}$ followed by @ $3.1251 \mathrm{ha}^{-1}$ during the both seasons in grape vine against all type of weed with no phytotoxic effect on grape vine even at double dose of application.

\section{References}

Aulakh, P. S., 1999, Chemical weed control in Perlette grape cuttings. Progressive Horticulture. 31: 68-69.

Bajwa, G. S., Brar, S. S., Minhas, P. P. S. and Cheema S. S. 1992, Efficacy of herbicides in controlling weeds in vineyards. Proceedings of the International Symposium on Recent Advances in Viticulture and Oenology, pp. 289-293.14-17 Feb., 1992. Hyderabad

Bajwa, G. S., Bal, J. S., Brar, S. S. and Minhas, P. P. S., 1989, Studies on the economic use of herbicides in vineyards. Paper presented in the First International Symposium on Horticultural Economics in Developing Countries. July16-23, pp. 11.Alemaya (Ethopia).

Bajwa, G. S., Brar, S. S., Bal, J. S. and Minhas, P. P. S., 1993, Efficacy of various herbicide to control weeds in the vineyards.Proceedings of International Symposium, Indian Society of Weed Science, Vol-III pp. 
222-224. 18-20, Nov., 1993. Hisar.

Bajwa, G. S., Brar, S. S., Bal, J. S. and Minhas, P. P. S., 1997, Studies on weed control in vineyards under different agro climatic conditions of Punjab. Indian Journal of Horticulture. 54: 5052.

Bajwa G. S. 1990. Weed management in vineyards. Paper presented in the National Seminar cum Workshop on Grapes for North west Plains p. 65. Punjab Agricultural University, Ludhiana.

Chanana, Y. B., Bajwa, G. S. and Hillon, B. S., 1975,Controlling weeds in young grapes. Progressive Farming. 12: 11.

Gaziev M. T and Voevodin, A.. V., 1985, Efficacy of herbicides applied to grapevines. Zashehita Rastenic.3: 24.

Hebbethwaite, J. F. and Schepens, G. R., 1986, Efficacy of glyphosate in viticulture. Horticultural Abstracts, 56: 170.

Hussain, S., Ramzan, M., Akhter, M. and Aslam, M., 2008, Weed management in direct seeded rice. Journal of Animal and Plant Science. 18 (2-3):86-88.
Krishna Sastry, K. S., Boraiah, G., Govindu, H. C. and Khakleel, T. F., 1980, Weeds of Karnataka, pp. 359. UAS Text book Series No.2. University of Agricultural Sciences, Bangalore.

Mani, M., Balasubramaniam, S. and Duraipandan, A. 1976, Effect of certain herbicides in the control of nut grass in vineyards. Indian Journal Plant Protection, 4:123-124.

Muniyappa, T. V. and Prathibha, N. C., 1993, Effect of herbicides on the control of Oxalis latifolia in grape garden. Proceedings of International Symposium. Indian Society of Weed Science, Vol. III pp. 275-276. 18-20, November, 1993. Hisar.

Rekha, K. B., Raju, M. S., and Reddy M. D., 2002,Effect of herbicides in transplanted rice. Indian Journal of Weed Science 34(1-2): 123- 125.

Stevenson, D. S., Neilson, G. I. U. and Corudsen, A., 1986, The effect of woven plastic mulch, herbicides, grass sod and nitrogen on field irrigation. Horticulture

Science 21: 439-441.

\section{How to cite this article:}

Nooli. S. S., M. B. Patil and Nilesh Shiragavi. 2020. Bioefficacy and Phytotoxicity of Oxyfluorfen $2.5 \%$ + Glyphosate $41 \%$ SC (Ready mix) against Weed Complex in Grape. Int.J.Curr.Microbiol.App.Sci. 9(07): 290-300. doi: https://doi.org/10.20546/ijcmas.2020.907.031 